\title{
All-Optical Flip-Flops using Electrically Pumped Microdisk Lasers Integrated on Silicon
}

\author{
K. Huybrechts ${ }^{1}$, L. Liu ${ }^{1}$, R. Kumar ${ }^{1}$, T. Spuesens ${ }^{1}$, G. Roelkens ${ }^{1}$, E.-J. Geluk ${ }^{2}$, T. de Vries ${ }^{2}$, \\ P. Regreny ${ }^{3}$, D. Van Thourhout ${ }^{1}$, R. Baets ${ }^{1}$, G. Morthier ${ }^{1}$ \\ ${ }^{1}$ Department of Information Technology, Photonics Research Group, Ghent University - IMEC \\ Sint-Pietersnieuwstraat 41, B-9000 Ghent, Belgium \\ e-mail: morthier@intec.ugent.be \\ ${ }^{2}$ COBRA Research Institute, Technische Universiteit Eindhoven, \\ Postbus 513, 5600 MB Eindhoven, The Netherlands \\ ${ }^{3}$ Université de Lyon, Institut des Nanotechnologies de Lyon \\ INL-UMR5270, CNRS, Ecole Centrale de Lyon, Ecully, F-69134, France
}

\begin{abstract}
We demonstrate flip-flop operation using the directional bistability in ultra-small microdisks $(7.5 \mu \mathrm{m}$ diameter) heterogeneously bonded on a silicon chip. The pulse energies are only $1.8 \mathrm{fJ}$ and the bias current is $3.5 \mathrm{~mA}$.
\end{abstract}

Index Terms - Microdisk lasers, All-optical flip-flops, Heterogeneous integration

\section{INTRODUCTION}

A LL-OPTICAL flip-flops are considered potential elements in all-optical packet switching schemes in order to cope with the massive bandwidth requirements resulting from the huge growth of upcoming telecommunication services [1-2]. They are of great importance in realizing such transparent packet/burst switches because they can act as optical memory elements and temporarily store the header information of data packets.

Several concepts for all-optical flip-flops have been proposed so far, but most of them require multiple active sections which results in a difficult integration and large power consumption [3-4]. One of the most well-known flipflop concepts in literature is composed of two coupled ring lasers that force each other to operate in a unidirectional operation either in a clockwise or a counterclockwise mode [5]. Using the non-linear gain suppression effect in such structures, it is possible to obtain this unidirectional regime also in a single ring or disk laser [6]. By injecting an optical pulse, one can switch between the two directional states and obtain flip-flop operation [7].

In this article we discuss recently obtained experimental results of all-optical flip-flop operation in a single microdisk

This work was supported by the European FP7 ICT-projects HISTORIC, WADIMOS and PhotonFAB, the Belgian Fund for Scientific Research Flanders (FWO), and the IAP-project 'Photonics@be'. The work of K.H. and T.S. is supported by the Institute for the Promotion of Innovation through Science and Technology (IWT) under a specialization grant.

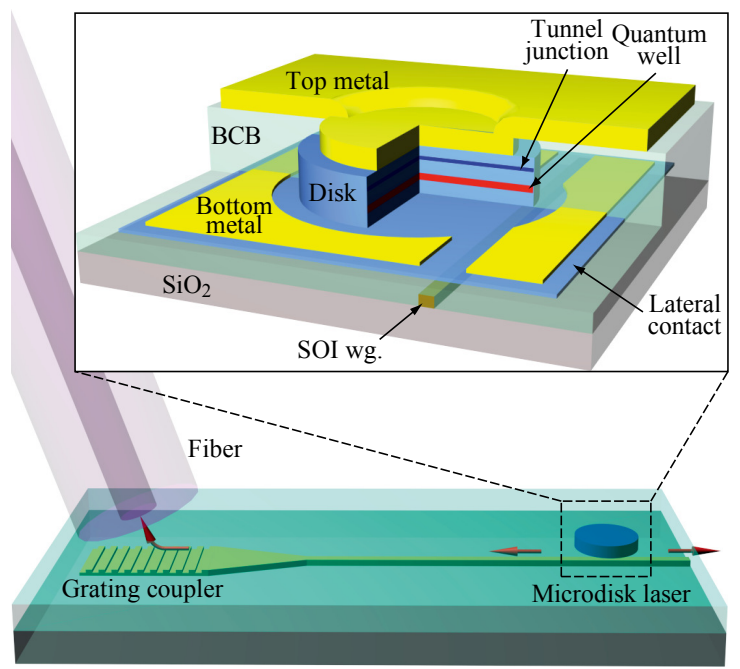

Fig. 1. Schematic of the fabricated structure: the microdisk laser is bonded on top of a silicon waveguide with grating couplers (from [10])

laser heterogeneously bonded on a silicon chip. The ultrasmall dimensions of the device $(7.5 \mu \mathrm{m}$ diameter) suggest a large potential for a dense integration. Moreover, the energy consumption is very low with a bias current of only $3.5 \mathrm{~mA}$ and pulse energies of $1.8 \mathrm{fJ}$.

\section{DeVice Structure}

A schematic of the fabricated structure is depicted in Fig. 1. The microdisk structure is heterogeneously bonded on a SOI chip through an adhesive die-to-wafer bonding [8]. The bonding agent is DVS-BCB (divinylsiloxanebenzocyclobutene). When current is injected through the top metal contact, the laser will start lasing in the whispering gallery mode. The laser light is evanescently coupled to a silicon waveguide underneath. Grating couplers on both sides of the waveguide allow an efficient output coupling to optical fibers. The SOI wafers were fabricated through a MPW- 
approach by the Silicon Photonics platform ePIXfab [9].

In order to obtain unidirectional operation, it is necessary to reduce the sidewall roughness and increase the photon density in the active layer. A low sidewall surface roughness was obtained by using an optimized etch process. To achieve a large internal power density, we introduced an additional heat sink which improved the thermal roll-over by a factor of two compared to previous designs. Using an improved wafer growth technique, the tunnel junction was placed on top of the microdisk to decrease absorption losses. A more detailed explanation of the device fabrication can be found in [10]

\section{EXPERIMENTAL RESULTS}

We measure the light-current (LI) characteristic simultaneously on both sides of the waveguide (see Fig. 2). We can see that the unidirectional regime starts at $1.7 \mathrm{~mA}$. The lasing threshold is at $0.33 \mathrm{~mA}$ and at low currents we observe a bidirectional regime where the two lasing directions are equally present. To avoid self-switching due to noise, we work at a bias current of $3.5 \mathrm{~mA}$ for our experiments. After taking the losses from the grating coupler into account, we receive an output power of $20 \mu \mathrm{W}$ in the silicon waveguide.

When the laser is in the unidirectional regime, the laser operates either in the clockwise or counterclockwise direction. We can switch between the two directions through injectionlocking with optical pulses. Figure 3 shows the typical time dependence of the on- and off-switching of a laser mode measured at one side of the waveguide. Because the disk is only coupled to a single straight waveguide, it is not possible to separate the switch pulses and the laser signal. Therefore the switch pulses always cover the transient of the microdisk signal, making it difficult to measure the exact switching times. The switch-off times were found to be as low as $60 \mathrm{ps}$. The pulse energies for switching are very low: all-optical flipflop operation was observed using pulses of only $1.8 \mathrm{fJ}$ (after normalizing with the losses from the grating coupler).

Besides flip-flop operation, we demonstrated also alloptical gates at $10 \mathrm{GHz}$ using pump-probe experiments. The disks did not have to be biased for this purpose.

The microdisks offer also great potential to implement various other optical processing functions and can be especially interesting for advanced all-optical logic, shift registers and Dflip-flops by a combination of several disks.

\section{CONCLUSION}

All-optical flip-flop operation is demonstrated in ultra-small microdisk lasers with diameters of only $7.5 \mu \mathrm{m}$. The disks are bonded on a SOI chip and record-low pulse energies of $1.8 \mathrm{fJ}$ can be used to switch between the clockwise and counterclockwise lasing mode. The device is promising for more advanced optical signal processing functions (eg. shift registers, all-optical logic). At the conference, results on new optimized devices will also be presented.

\section{REFERENCES}

[1] R. Van Caenegem, J. A. Martinez, D. Colle, M. Pickavet, P. Demeester, F. Ramos, and J. Marti, "From IP over WDM to all-optical packet

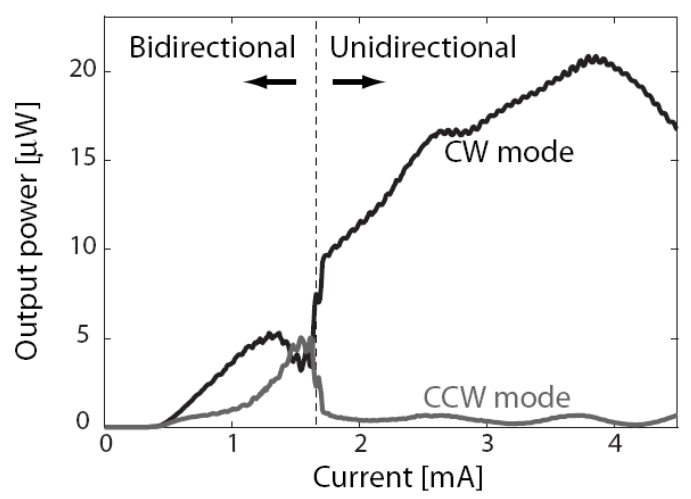

Fig. 2. Light-current (LI) characteristic (measured at the two ends of the waveguide) indicates that the unidirectional operation starts at $1.7 \mathrm{~mA}$.

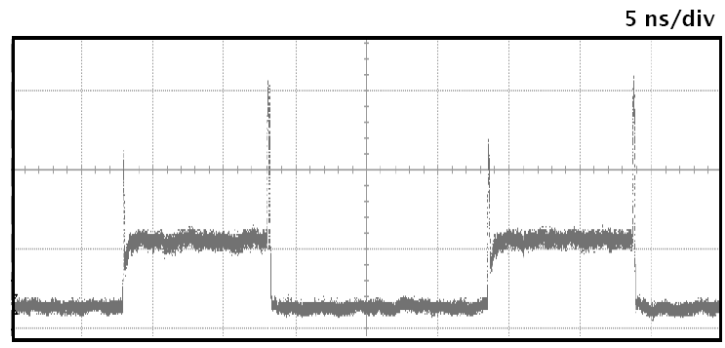

Fig. 3. Experimental demonstration of high-speed flip-flop operation in a single microdisk laser.

switching: Economical view," Journal of Lightwave Technology, 24, 1638-1645 (2006).

[2] H. J. S. Dorren, M. T. Hill, Y. Liu, N. Calabretta, A. Srivatsa, F. M. Huijskens, H. de Waardt, and G. D. Khoe, "Optical packet switching and buffering by using all-optical signal processing methods," Journal of Lightwave Technology, 21, 2-12 (2003).

[3] Malacarne, A., Wang, J., Zhang, I, Das Barman, A, Berrettini, G., Poti, L., Bogoni, A., 20-ps transition time all-optical SOA-based flip-flop used for photonic $10 \mathrm{~Gb} / \mathrm{s}$ switching operation without any bit loss, IEEE Journ. Sel. Top. Quant. El., 14, 808-815 (2008).

[4] M. T. Hill, H. de Waardt, G. D. Khoe, and H. J. S. Dorren, "All-optical flip-flop based on coupled laser diodes," Journal of Quantum Electronics, vol. 37, pp. 405-413, Mar 2001.

[5] M. Hill, H. J. S. Dorren, T. de Vries, X. J. M. Leijtens, J. H. den Besten, B. Smalbrugge, Y. S. Oei, H. Binsma, G. D. Khoe, and M. K. Smit, "A fast low-power optical memory based on coupled micro-ring lasers," Nature, 432, 206-209 (2004).

[6] M. Sorel, G. Giuliani, A. Scire, R. Miglierina, S. Donati, and P. J. R. Laybourn, "Operating regimes of GaAs-AlGaAs semiconductor ring lasers: Experiment and model," IEEE Journal of Quantum Electronics, 39, 1187-1195 (2003).

[7] A. Trita, G. Mezosi, F. Bragheri, Y. Jin, S. Furst, W. Elsasser, I. Cristiani, M. Sorel, and G. Giuliani, "Dynamic operation of all-optical flip-flop based on a monolithic semiconductor ring laser," in 34th European Conference on Optical Communication, 2008 (ECOC 2008).

[8] G. Roelkens, J. Van campenhout, J. Brouckaert, D. Van Thourhout, R. Baets, P. Romeo, P. Regreny, A. Kazmierczak, C. Seassal, X. Letartre, G. Hollinger, J.M. Fedeli, L. Di Cioccio, C. Lagahe-Blanchard, "III-V/Si photonics by die to wafer bonding," Materials Today 10(7), p.36-43 (2007)

[9] Silicon Photonics Platform ePIXfab: www.epixfab.eu

[10] L. Liu, R. Kumar, K. Huybrechts, T. Spuesens, G. Roelkens, E-J. Geluk, T. de Vries, P. Regreny, D. Van Thourhout, R. Baets, G. Morthier, An ultra-small, low-power all-optical flip-flop memory on a silicon chip, Nature Photonics, 4(3), p.182-187 (2010). 